\title{
Gas-water stratified flow patterns from electromagnetic tomography
}

\author{
Wang Xiaoxing and Wu Xiling* \\ School of Resources and Information Technology, China University of Petroleum, Beijing 102249, China
}

\begin{abstract}
Because of gravitational differentiation of multi-phase fluids, gas-water flow is usually stratified in highly inclined or horizontal gas wells. By using electrode arrays to scan flowing fluids, electromagnetic tomography can identify the flow patterns of mixed fluid from the different electrical properties of gas and water. The responses for different gas-water interface locations were calculated and then physical measurements were undertaken. We compared the results of the numerical simulation with the experimental data, and found that the response characteristics were consistent in the circumstances of uniform physical fields and stratified flows. By analyzing the signal characteristics, it is found that, with the change of the interface location, the response curves showed "steps" whose position and width were decided by the location of fluid interface. The measurement accuracy of this method depended on the vertical distance between adjacent electrodes. The results showed that computer simulation can simulate the measurement of the electromagnetic tomography accurately, so the physical experiment can be replaced.
\end{abstract}

Key words: Electromagnetic tomography, stratified flow, gas-water, numerical simulation, physical experiment

\section{Introduction}

Because of gravitational differentiation of multi-phase fluids, gas-water flow is usually stratified in highly inclined or horizontal gas wells. Conventional flow measuring techniques are linear methods that average the local values and cannot give accurate descriptions of the fluid distribution in the well (Wu, 2004).

In order to obtain satisfactory results, the Schlumberger Company developed a new production logging instrument for horizontal wells-Flow Scanner, which has four miniature spinners, five electrical probes, and five optical probes designed to measure the velocity profile of the borehole fluid, and the fraction of water and gas present in an interval of pipe (the local water and gas holdups). The electrical probes measure fluid impedance to distinguish hydrocarbons and water and the optical probes GHOST (Gas Hold Optical Sensor Tool) are used to identify oil and gas from their refractive indices (Wang et al, 2007). The CAT (Capacitance Array Tool) developed by the Sondex Company has an array of 12 capacitance sensors placed on the backup arm of the MAC (Multi-Arm Caliper) device, which can distinguish oil, gas, and water according to their dielectric constants around the probes (Ni and Zheng, 2004). The MCFM (MultiCapacitance Flow Meter) developed by the Baker Atlas Company works on a similar principle. It is clear that the present tools just measure the local fluid without a whole

*Corresponding author. email: wuxiling@sina.com

Received August 18, 2008 scanning measurement.

In the recent years, flow imaging techniques, as a nonlinear measuring method, have made rapid progress with the development of measurement science and computer techniques (Peyton et al, 1999; Bolton et al, 2007; Tan et al, 2007; Yang and Liu, 2000; Li and Huang, 2000; Bolton and Primrose, 2005). For the special circumstance of multi-phase flow in the well, Wu et al $(1999 ; 2000 ; 2008)$ introduced flow imaging techniques to the well logging field and proposed an electromagnetic tomography method for the multi-phase flow according to the differences of electrical properties among oil, gas, and water (Zhao and $\mathrm{Wu}, 2003$; Zhao et al, 2007). Based on the previous work, we used electromagnetic tomography for the gas-water stratified flow, and studied the characteristics and changing rules of the measuring responses by numerical simulation and physical experiments.

\section{Measuring model}

The electromagnetic tomography sensor is designed as shown in Fig. 1 and Fig. 2. A composite structure of the sensor consists of three layers of ringed electrode arrays. The first and the third layers are the shielding electrode layers, and the middle one is the main electrode layer. Sixteen electrodes are located around the circle of the inner wall in each layer. In order to reduce the influence of the steel casing on the measuring signals, electrode arrays are installed on a corrosion-resistant and insulating Teflon bush.

During the measurement, we chose an electrode in the main electrode layer to emit signal, and two focusing electrodes and two shielding electrodes close to it on the 


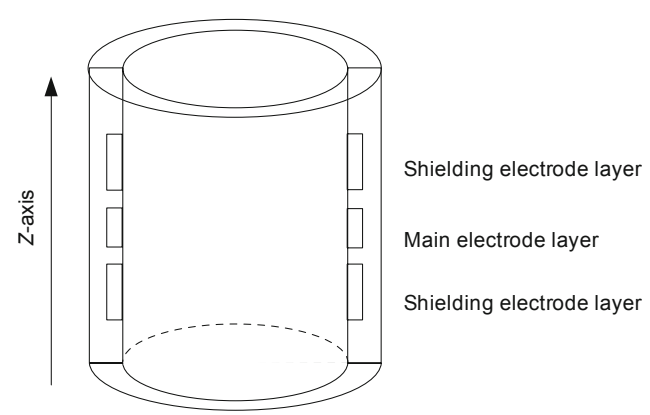

Fig. 1 Schematic diagram of electrode arrays

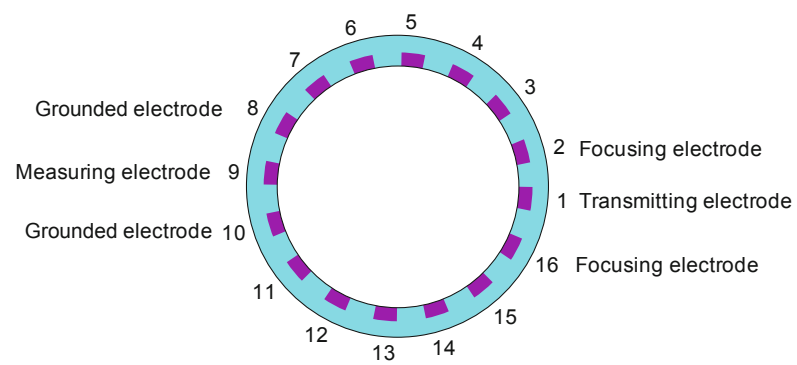

Fig. 2 Section of the main electrode layer and measuring mode

left and right, above and below emitted the same signals. Then, the other electrodes in the main electrode layer were switched to be measuring electrodes in turn, and received the responses to the fluid medium in different directions. The four electrodes close to the measuring electrode are all grounded. Because of 11 measuring data for one transmitting electrode, the number of measuring data in a period is $16 \times(16-5)=176$.

Zhao (2002) proved that the electric potential near the main electrodes is uniform in the $Z$-axis by analyzing the distribution of the 3-D electromagnetic field. Therefore, 3-D simulation can be transformed to 2-D, which can simplify the problem. When the frequency of the electromagnetic wave is lower than $3 \mathrm{MHz}$, it can be proved that the electromagnetic field is a near field in the range of pipeline diameter and is a time-varying field under quasi-static conditions (Shen, 2006; Zou, 2002). An excitation signal with a common harmonic voltage is emitted. Ignoring the coupling of electric and magnetic fields, the definite problem of the 2-D electromagnetic field can be described as follows according to Maxwell's equations:

$$
\left\{\begin{array}{lc}
-\nabla \cdot(\varepsilon \nabla \phi)+\frac{j}{\omega} \nabla \cdot(\sigma \nabla \phi)=0 \quad(\phi \in D) \\
\phi=1 & \left(\Gamma_{1}: \phi \in \text { source electrode }\right) \\
\phi=0 & \left(\Gamma_{2}: \phi \in \text { earthed electrode }\right)
\end{array}\right.
$$

where, $D$ is the area to be solved; $\Gamma_{1}$ is the boundary of the transmitting and focusing electrodes; $\Gamma_{2}$ is the boundary of the grounding electrodes.

\section{Simulation of the measuring responses}

According to Fig. 2, a sine signal with the voltage of $1 \mathrm{~V}$ and the frequency of $3 \mathrm{MHz}$ is applied to the transmitting and focusing electrodes. The real and imaginary parts of the complex number result can be obtained by FEM (Finite Element Method). Then, the amplitude and phase can be calculated as follows:

$$
\begin{aligned}
& a(r)^{2}=Q_{\mathrm{r}}(r)^{2}+Q_{\mathrm{j}}(r)^{2} \\
& \tan (\phi(r))=Q_{\mathrm{j}}(r) / Q_{\mathrm{r}}(r)
\end{aligned}
$$

where, $a(r)$ is amplitude, $\phi(r)$ is phase; $Q_{\mathrm{r}}(r)$ and $Q_{\mathrm{j}}(r)$ are real part and imaginary part, respectively.

Fig. 3 shows the potential distribution when the first electrode is the main transmitting electrode and the fifth electrode is the measuring electrode. The gas-water (blue is the saline water of $1 \Omega \cdot \mathrm{m}$, white is air) interface lies between the fifteenth and the sixteenth electrodes.

It is clear that the distribution of media affects the potential distribution strongly. In the air region, equipotential lines are denser which means the potential changes faster. The values of the real part are greater than those of the imaginary part. The potential distributions corresponding to other combinations of measuring electrodes are similar.

As the changes of the amplitude are more regular, we mainly analyze the response curves of the amplitude. Fig. 4 shows a period of the response curve (176 measured values) of the model in Fig. 3. The main transmitting electrode rotates from the thirteenth electrode counterclockwise. For convenient comparison, the response curves of uniform fields of air and saline water are displayed together.
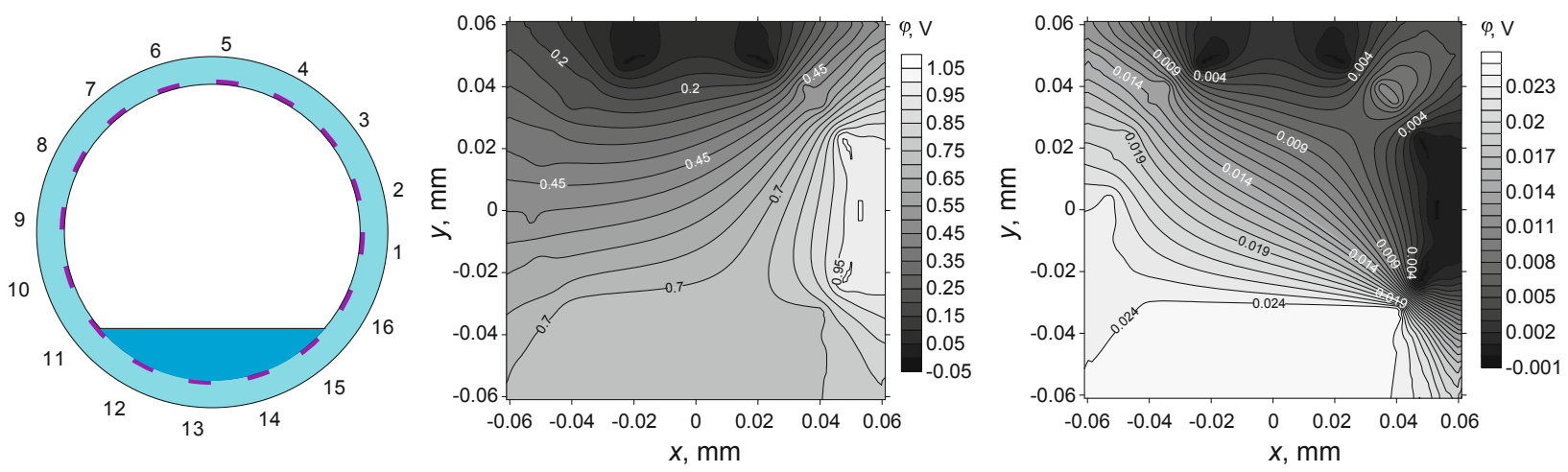

Fig. 3 Potential distribution of real and imaginary parts of gas-water stratified flow 


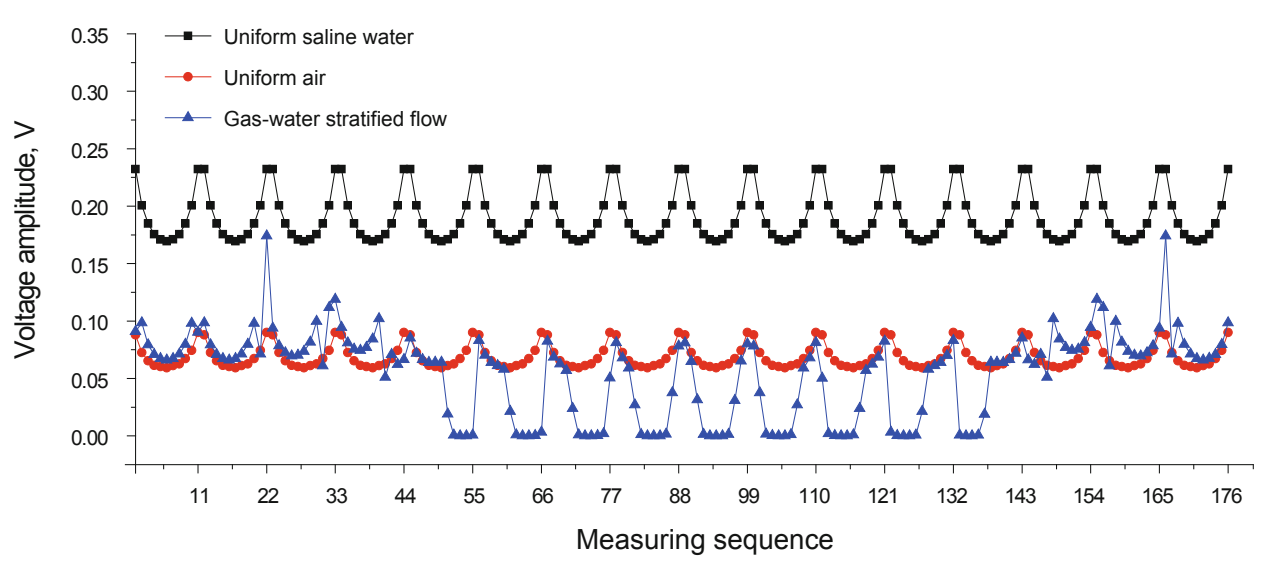

Fig. 4 Simulated response curves of gas-water stratified flow (five electrodes in the water)

Fig. 4 indicates that because of the same medium distribution of the uniform field, the response curves of the 16 electrodes have the same "U" shape according to the different distances between the transmitting electrode and measuring electrode. The lowest point corresponds to the value of the measuring electrode opposite to the transmitting one. Moreover, the values of the saline water are greater than those of the air.

For the measurement of a single transmitting electrode (11 measured values), when the medium in the pipeline is stratified, wherever the transmitting and focusing electrodes are, if the measuring electrode is in the air, the measured values are always between those of the two uniform fields. However, if the transmitting and focusing electrodes are in the air and the measuring electrode is in the water, the measured values are almost zero, which makes the response curve show a "step", whose width depends on the number of the measuring electrodes in the water. Many "steps" in the response curves of a single transmitting electrode compose the "step" in the curve of a period (176 measured values). The whole width depends on the number of the electrodes in the air of all 16 electrodes.

\section{Physical modeling}

Air and saline water (resistivity is $1 \Omega \cdot \mathrm{m}$ ) were selected for the static physical model experiment. The measurement system consisted of a measurement sensor, control circuit, simple flow simulator, and an HP8753C network analyzer which was used to create and measure the electromagnetic fields. As the network analyzer provides a constant power, the attenuation values of power are measured. The voltage amplitude can be calculated from the attenuation values of power.

$$
F=10 \lg \frac{P_{\text {output }}}{P_{\text {input }}}=10 \lg \frac{U_{\text {output }}^{2} / R_{\text {output }}}{U_{\text {input }}^{2} / R_{\text {input }}}
$$

where, $F$ is the attenuation values of power.

At first, the experiment was performed with either air or saline water. Fig. 5 shows the comparison of the experimental and simulated data. Because of the repetition of the curve characteristics with one-phase fluid, only 22 measured values when the first and the second electrodes were the transmitting electrodes are presented as an example. Fig. 6 shows the comparison of the simulated data and experimental data of the gas-water stratified flow model in Fig. 3. Good consistencies were shown in Fig. 5 and Fig. 6, which proved the accuracy of the numerical simulation.

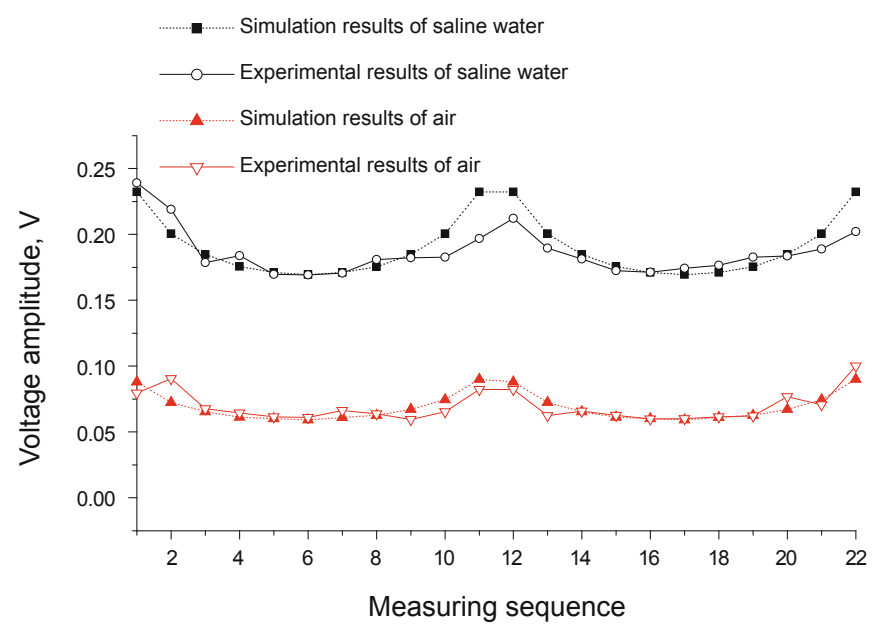

Fig. 5 Comparison of the experimental and simulated data with a singlephase fluid

\section{Analysis of signal characteristics}

Because the computer simulation can simulate the measurement of the electromagnetic tomography accurately, the physical experiment can be replaced. Fig. 7 shows the response curves when the gas-water interface is near the top of the pipeline, taking the interface between the first electrode and the second electrode for example. When the transmitting and focusing electrodes are in the air and the measuring electrode is in the water, the "step" still appears. The differences from the response curve of the stratified flow in Fig. 4 are that the numbers of zero values are more which means the width of the "step" is larger because of more 


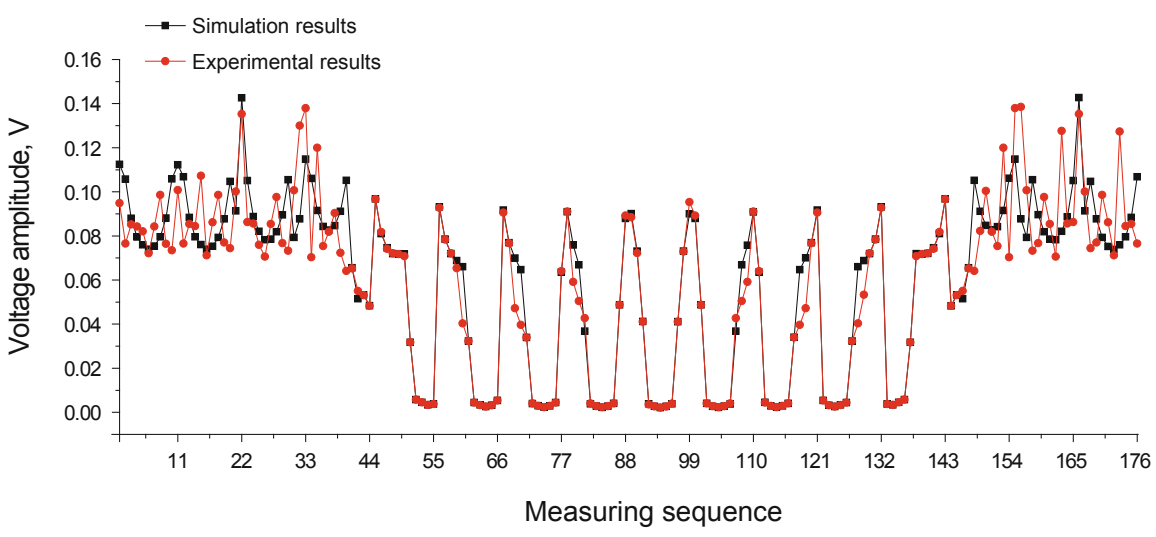

Fig. 6 Comparison of the experimental and simulated data from gas-water stratified flow

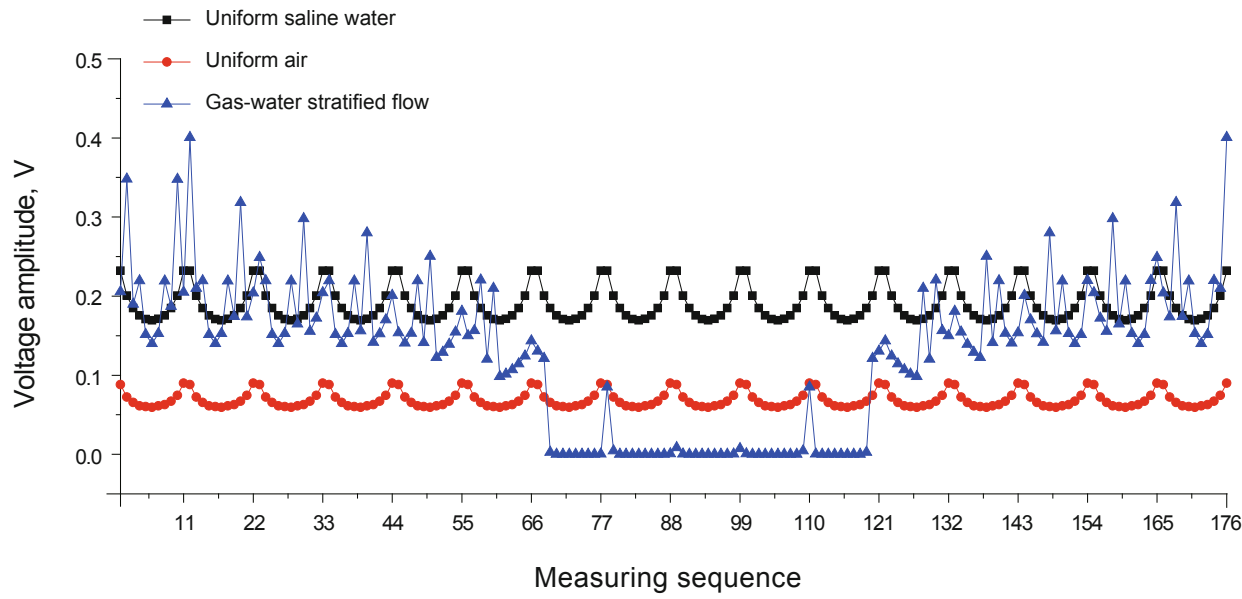

Fig. 7 Simulated response curves of gas-water stratified flow (nine electrodes in the water)

electrodes in the water. The width of the whole "step" in a period of measurement (176 values) decreases because fewer electrodes are in the air.

In order to study the measurement accuracy, the response curves for different locations of interface were simulated. The results showed that if the gas-water interface was between the same electrodes but at different places, the shapes of the curves were exactly the same. With the increase of the water content, the measured values became greater. Therefore, according to the curve characteristics, the measurement accuracy, namely the discernible height of the stratified flow, is the vertical distance between two adjacent electrodes above and below.

When the equipment was placed in the well, the relative position of the equipment and the gas-water interface was unknown, and the interface may be slanting. In this situation, the characteristics of the response curves still met the abovementioned rules, which were also proved by numerical simulation.

\section{Conclusions}

1) With change of the interface location, the response curves showed "steps" whose position and width were decided by the places where the transmitting, focusing and measuring electrodes are.

2) The measurement accuracy, namely the discernible height of the stratified flow, is the vertical distance between two adjacent electrodes above and below according to the curve characteristics.

3) Electromagnetic tomography can identify gas-water stratified flow and provides a new method of flow profile measurement. Computer simulation can simulate the measurement of the electromagnetic tomography accurately, so the physical experiment can be replaced.

\section{References}

Bolton G T and Primrose K M. An overview of electrical tomographic measurements in pharmaceutical and related application areas. AAPS PharmSciTech. 2005. 6(2): E137-E143

Bolton G T, Bennett M, Wang M, et al. Development of an electrical tomographic system for operation in a remote, acidic and radioactive environment. Chemical Engineering Journal. 2007. 130(2-3): 165169

Li H Q and Huang Z Y. Special Detection Technology and Application. Hangzhou: Zhejiang University Press. 2000. 1-9 (in Chinese)

Ni G J and Zheng X X. Application of a new model multiple phase holdup logging instrument in horizontal well—Capacitance array 
multiple phase holdup logging instrument (CAT). Well Testing. 2004. 13(4): 86-89 (in Chinese)

Peyton A J, Beck M S, Borges A R, et al. Development of electromagnetic tomography (EMT) for industrial applications. In: Proceedings of the World Congress on Industrial Process Tomography. Buxton: Greater Manchester. 1999. 306-312

Shen X N. Electromagnetic Fields and Electromagnetic Waves. Beijing: Science Press. 2006. 78-81 (in Chinese)

Tan C, Dong F and Wu M M. Identification of gas/liquid two-phase flow regime through ERT-based measurement and feature extraction. Flow Measurement and Instrumentation. 2007. 18(5-6): 255-261

Wang G F, Wu L and Liu T. Flow Scanner: A new flow tool for production logging in horizontal wells. Petroleum Instruments. 2007. 21(2): 33-36 (in Chinese)

Wu X L. Petroleum Production Logging Principles. Beijing: Higher Education Press. 2004. 213-236 (in Chinese)

Wu X L, Jing Y Q and Wu S Q. Electromagnetic imaging logging method in multiphase pipe flow. Chinese Journal of Geophysics. 1999. 42(4): 557-563 (in Chinese)

Wu X L, Wang X X, Zhao Y W, et al. Flow imaging method of electromagnetic measurement in well logging. Science in China, Series D: Earth Sciences. 2008. 38(S2): 161-165

Wu X L, Zhao L and Liu D J. A fundamental study on electromagnetic wave imaging logging in multiphase flow. Petroleum Exploration and Development. 2000. 27(2): 79-82 (in Chinese)

Yang W Q and Liu S. Role of tomography in gas/solids flow measurement. Flow Measurement and Instrumentation. 2000. 11(3): 237-244

Zhao L. Study on flow imaging logging using electromagnetic wave. Ph.D. Thesis. Beijing: China University of Petroleum. 2002 (in Chinese)

Zhao L and Wu X L. Calculation of sensitivity field for electromagnetic tomography in multiphase flow well logging. Chinese Journal of Geophysics. 2003. 46(6): 870-874 (in Chinese)

Zhao Y W, Wu X L and Wang X X. Simulation of sensitivity field for electromagnetic tomography in multiphase flow well logging. Chinese Journal of Geophysics. 2007. 50(3): 811-816 (in Chinese)

Zou L. Modeling of electrical resistance tomography. Master Degree Thesis. Beijing: Tsinghua University. 2002 (in Chinese)

(Edited by Hao Jie) 